\title{
ORGANIZAÇÃO DO XXIV CONGRESSO BRASILEIRO DE ENFERMAGEM
}

\section{PRESIDENTES-DE-HONRA}

\author{
MARECHAL EMILIO GARRASTAZU MEDICI \\ Presidente da República \\ DR. JARBAS GONCALVES PASSARINHO \\ Ministro da Educação e Cultura \\ DR. RONDON PACHECO \\ Governador do Estado de Minas Gerais \\ I)R. FERNANDO MEGRE VELOSO \\ Secretário de Estado da Saúde
}

\section{MEMBROS DE HONRA}

Prof. Marcelo de Vasconcelos Coelho

Reitor da Universidade Federal de Minas Gerais

Prof. Marino Mendes Campos

Vice-Reitor da Universidade Federal de Minas Gerais

Dom João de Rezende Costa

Arcebispo Metropolitano de Belo Horizonte

Dom Serafim Fernandes de Araújo

Reitor da Universidade Católica de Minas Gerais

Dr. Oswaldo Pieruccetti

Prefeito Municipal de Belo Horizonte

Dr. Caio Benjamim Dias

Secretário de Estado da Educação

Dr. Abílio Machado

Secretário de Estado do Governo 
Dr. Paulo Campos Guimarães

Diretor da Imprensa Oficial

Dr. Francisco Neves

Secretário Municipal de Saúde

Doutora Suzana Gonçalves

Chefe da Assessoria de Programas da CAPES

Prof. Eduardo Rodrigues Affonso de Moraes

Secretário do Conselho Universitário

Dr. Celso Barroso Leite

Diretor-Executivo da CAPES

Senhora Maria Aparecida Pourchet Campos

Conselheira da CAPES

Dr. Nelson Afonso do Valle Silva

Secretário-Geral da Fundação ABIF

Prof. Raimundo José de Castro Muniz Aragão

Presidente da Fundação ABIF

Dr. Décio Marri

Secretário-Geral da UFMG

Prof. Fábio do Nascimento Moura

Chefe do Gabinete do Magnífico Reitor da UFMG

Dr. Guido Antônio de Caux

Diretor da Divisão de Pessoal da UFMG

Prof. Hélio Pontes

Diretor da Divisão de Planejamento da UFMG

Prof. Miguel de Souza

Prefeito da Cidade Universitária da UFMG

General de Brigada Everaldo José da Silva

Comandante da ID/4

Major Brigadeiro Roberto Julião Cavalcanti de Lima

Comandante da Base Aérea de Belo Horizonte

Coronel Luiz Nunes Neto

Comandante-Geral da Polícia Militar de Minas Gerais

Coronel Idimar Vilas Boas

Chefe do Estado Maior da Polícia Militar de Minas Gerais

Coronel Odelmo Teixeira Costa

Secretário de Estado da Segurança Pública de Minas Gerais

Coronel Vicente Gomes da Mota

Chefe da Casa Militar do Governo do Estado de Minas Gerais. 


\section{HOMENAGENS ESPECIAIS}

Prof. Hélio Martins de Araújo Costa

Diretor do Conselho de Pesquisa da UFMG

Prof. José Eduardo da Fonseca

Diretor do Conselho de Extensão da UFMG

Prof. Leônidas Machado Magalhães

Diretor do Conselho de Pós-Graduação da UFMG

Prof. Oder José dos Santos

Diretor dó Conselho de Graduação da UFMG

Prof. Adônis Martins Moreira

Secretário de Coordenação Administrativa da UFMG

Prof. Haroldo de Almeida Mattos

Diretor da Escola de Belas Artes da UFMG

Prof. José Pinto Machado

Diretor da Faculdade de Medicina da UFMG

Dr. João Marques

Diretor da Imprensa Universitária da UFMG

Dr. Sebastião Rios Júnior

Diretor da Divisão de Contabilidade da UFMG

Prof. Armando Ribeiro dos Santos - UFMG

Prof. Osny Pereira

Presidente do Conselho Diretor da Assistência aos Universitários "Mendes Pimentel"

Prof..$^{a}$ Marília Otoni da Silva Pereira

Diretora da COPERTIDE da UFMG

Prof. Plínio Carneiro

Chefe dos Serviços das Relações Universitárias da UFMG

Prof. Jayme Neves

Faculdade de Medicina da UFMG

Prof. Washington Luiz Tafuri

I.C.B. - UFMG

Prof. David Márcio dos Santos - UFMG

Prof. Eduardo de Paula

Escola de Belas Artes da UFMG

Senhora Carmem de Carvalho Lena - UFMG

Dr. Christiano Lopes de Rezende

Diretor do Hospital Júlia Kubitschek 
Dr. Eduardo Cisalpino

Diretor do I.C.B. da UFMG

Prof. Roberto Carneiro - UFMG

Dr. Roberto Leão Lessa

Diretor da Divisão de Patrimônio da UFMG

Dr. David Hazan

Diretor da D.O.P.S.

Dr. Gilberto M. Pessoa

Chefe da Agência do SNI em Belo Horizonte

PRESIDENTE DO CONGRESSO

Amália Corrêa de Carvalho

Presidente da Associação Brasileira de Enfermagem

COMISSÃO DE TEMAS

Anayde Corrêa de Carvalho, Coordenadora

Dinéa Tognini

Rosa Aparecida Pimenta de Castro

COMISSÃO DE RECOMENDAÇÕES

Dra. Glete de Alcântara, Coordenadora

Maria Francisca Rangel de Jesus Barros

Celina Arruda Camargo

\section{COMISSÃO EXECUTIVA}

Izaltina Goulart de Azevedo, Presidente

Carmelita Pinto Rabelo, Secretária

Clélia Luiza Gonçalves Pinto, Tesoureira

\section{SUBCOMISSÕES}

\section{SUBCOMISSÃO DE FINANÇAS}

Clélia Luiza Gonçalves Pinto, Coordenadora Dilza de Brito Guimarães 


\section{SUBCOMISSÃO DE DIVULGAÇÃO}

Maria José Silva, Coordenadora

Aparecida Ferreira Moura

Irmã Hygina Bueno Bruzzi

Helena Pereira

Maria Zélia Carneiro

SUBCOMISSÃO DE REGISTRO

Maria Aparecida Sampaio, Coordenadora

Wilma Pinto Castro

Olga Shirley Carneiro Ricardo

Maria José Cruz Homem

Maria Barbosa

Lêda Carvalho Oliveira

\section{SUBCOMISSÃO SOCIAL}

Águida Stemler de Oliveira, Coordenadora Eni Enes Raquel de Oliveira

Lourdes Bueno Maia

Helena Pereira

Maria Lídia de Queiroz Ribeiro da Rocha

\section{SUBCOMISSÃO DE RECEPÇÃO}

Luzia Silva, Coordenadora

Maria Girlene Martins

Alzira Melo

Iara Bastos

Silvéria Rodrigues Ferreira

Adelina Gonçalves Sobrinho

Ana Lúcia de Faria

Maria das Graças Oliveira

Iracema Mamede

Maria da Conceição Teixeira

\section{SUBCOMISSÃO DE HOSPEDAGEM}

Maria do Rosário Melo, Coordenadora

Maria Army de Oliveira

Fabiantur Turismo 


\section{SUBCOMISSÃO DE MONITORIA}

Ana Maria Maroni Miranda, Coordenadora

Maria Bernardete Queiroga

Luciola Queiroga

Maria Lúcia Andrade Moreira

SUBCOMISSÃO DE PREPARO DE LOCAL

Izaltina Goulart de Azcvedo, Coordenadora

Carmelita Pinto Rabelo

Irmã Bernadete Luz

Iracy Ferreira da Silva

Nancy de Almeida Uchoa

\section{SECRETARIA}

Lygia Queiroz Guimarães

Elio Sobreiro

\section{MEMBROS COLABORADORES}

Dr.Luiz de Almeida, Chefe do Departamento Administrativo do Palácio do Governo do. Estado de Minas Gerais

Dra. Ester de Carvalho, Secretária do Sr. Governador do Estado

Dr. Roberto Mafra, Chefe do Departamento Administrativo da Secretaria de Estado da Saúde

Dr. Jaire Peres Vasconcelos - Gerente do Banco do Brasil S.A.

Escola de Enfermagem da UFMG

Faculdade de Comunicação da Universidade Católica de Minas Gerais

Comissão Organizadora do Sesquicentenário da Independência

Laboratórios Johnson \& Johnson do Brasil S.A.

Laboratórios Wintrop

Laboratórios Miles do Brasil

Cremer S.A.

Laboratórios Darrow

Laboratório Farmitália

Indústrias York S.A.

Laboratório Hoescht

Laboratórios Becton Dickinson 
Hospital das clínicas da UFMG

Fundação Benjamim Guimarães - Hospital da Baleia

Banco de Crédito Real de Minas Gerais S.A.

Banco do Brasil S.A.

Banco Mineiro do Oeste S.A.

Nativa Construções Elétricas S.A.

Vasp - Viação Aérea São Paulo

Varig S.A.

Cruzeiro do Sul - Serviços Aéreos

Fabiantur Turismo

Fábrica de Tecido Santa Cruz, de Juiz de Fora - MG

Tecidos Lima S.A.

Utramig. 


\section{T EM A R I O}

TEMA I:

Formação dos profissionais de enfermagem dos três níveis

TEMA II :

Exercício profissional

TEMA III:

Simpósio - A enfermeira e a comunidade

TEMA IV :

Recentes pesquisas no campo da enfermagem 